\title{
Relaxation of tracheal smooth muscle is impaired in innate airway hyperresponsiveness
}

\author{
F-X. Blanc* ${ }^{*}$ C. Coirault ${ }^{\#}$, P. Oliviero ${ }^{\#}$ and Y. Lecarpentier ${ }^{\top},+$
}

ABSTRACT: The current study was designed to determine whether the nonspecific in vivo airway hyperresponsiveness of the inbred Fisher F-344 rat strain is associated with impaired spontaneous relaxation of airway smooth muscle.

Strips of the posterior portion of the trachea from 10 adult Fisher and 10 adult Lewis rats were electrically stimulated at $\mathrm{pH} 7.4,2.5 \mathrm{mM} \mathrm{Ca}^{2+}$ concentration, at $37^{\circ} \mathrm{C}$. Both isotonic and isometric relaxations of tracheal smooth muscle (TSM) were investigated.

Half time for isotonic relaxation at preload was markedly prolonged in Fisher rats $(8.33 \pm 3.21 \mathrm{~s})$ compared with Lewis rats $(3.53 \pm 0.54 \mathrm{~s} ; \mathbf{p}<0.001)$. Maximum lengthening velocity at preload and peak rate of isometric tension decline were significantly decreased in Fisher rats compared with Lewis rats. The ratio of shortening velocity to lengthening velocity at preload, as well as the ratio of the isometric peak rates of tension development to tension decline were higher in Fisher rat TSM than in Lewis rat TSM. These differences were associated with a six-fold higher expression of myosin light chain kinase in Fisher rats than in Lewis rats.

In Fisher rats, these results suggest that innate airway hyperresponsiveness is associated with both a reduced level and a slower rate of TSM spontaneous relaxation, promoting maintenance of airway constriction.

KEYWORDS: Airways, hyperresponsiveness, isometry, isotonic, relaxation, smooth muscle

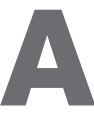

irway hyperresponsiveness (AHR), a cardinal feature of asthma, is usually defined as excessive narrowing of airways in response to nonspecific contractile agonists. Although it is clear that airway smooth muscle (ASM) is the key effector of excessive airway narrowing in asthma, it remains unclear whether or not AHR is attributable to ASM abnormality [1]. However, there is growing evidence that functional changes of ASM can result in increased ASM shortening capacity and velocity in hyperresponsive airways.

To study the direct contribution of ASM in the phenomenon of AHR, two complementary approaches are generally used in animals. First, various models dealing with passive or active sensitisation are used to investigate the acquired mechanisms involved in AHR. Secondly, models of spontaneous, nonspecific AHR can be used to study the inherited, genetically determined factors associated with AHR. Two rat strains have been extensively studied because of marked differences in their spontaneous airway responsiveness: the Fisher F-344 strain and the Lewis strain. When compared with the Lewis strain, the Fisher strain exhibited greater airway responsiveness to various inhaled contractile agonists. This relative AHR was manifested by a shift in the dose-response curve to aerosolised agonists in both spontaneously breathing [2-4] and mechanically ventilated animals [5]. Thus, the Fisher and Lewis model can be seen as a paradigm similar to asthmatic and normal humans [6]. It has been recently shown that, in vitro, electrically stimulated tracheal smooth muscle (TSM) of Fisher rats exhibited higher shortening capacity and velocity than in Lewis rats [7]. These mechanical differences were associated with differences in myosin crossbridge kinetics, i.e. shorter duration of crossbridge detachment and attachment in Fisher than Lewis rats, highlighting the crucial role of the crossbridge itself in the phenomenon of innate greater airway responsiveness of the Fisher rat strain [7].

However, ASM may contribute to AHR not only by its increased ability to shorten, but also by an impairment of its ability to relax [8]. Relaxation is
AFFILIATIONS

*Unité de Pneumologie, Service de Médecine Interne

'Service d'Explorations

Fonctionnelles Cardiovasculaires et Respiratoires, Centre Hospitalier Universitaire de Bicêtre, UFR Paris XI Assistance Publique, Hôpitaux de Paris, Le Kremlin Bicêtre, \#INSERM U974, Institut de Myologie, UPMC Université Paris VI, Paris, and

${ }^{+}$Centre de Recherche Clinique Centre Hospitalier de Meaux, Meaux, France.

CORRESPONDENCE

F-X. Blanc

Unité de Pneumologie, Service de Médecine Interne

Centre Hospitalier Universitaire de Bicêtre

78 rue du Général Leclerc 94275 Le Kremlin Bicêtre

France

E-mail: xavier.blanc@bct.aphp.fr

Received:

July 102008

Accepted after revision:

Jan 052009 
described as the process during which the muscle returns to its initial length and tension [9]. Spontaneous relaxation must be differentiated from pharmacological relaxation. The former occurs because of the attenuation or cessation of a contractile stimulus and consists of mechanical events driven by forces that bring the tissue to its original mechanical equilibrium [10]. The latter is induced by pharmacological agents (called bronchodilators) that act either directly on ASM or by inhibiting neural pathways [11]. While pharmacological relaxation has been extensively studied, almost exclusively in isometric preparations, spontaneous relaxation has been rarely investigated. In sensitised dogs, it has been shown that spontaneous isotonic relaxation of electrically stimulated bronchial smooth muscle was prolonged [12, 13], suggesting that factors operating during electrical stimulation might affect ASM relaxation and contribute to the AHR of sensitised tissue [10]. In spontaneous, inherited, genetically determined AHR, spontaneous relaxation remains to be investigated.

Therefore, the aim of the present study was to determine whether the nonspecific in vivo AHR of the Fisher F-344 rat strain was associated with differences in the intrinsic relaxation properties of electrically stimulated TSM when compared with Lewis rats. We tested the hypothesis that inherited genetically determined mechanical differences of the contraction process could be associated with differences in both isotonic and isometric spontaneous relaxation. We also investigated whether differences in isotonic or isometric relaxation of TSM were associated with differences in the tracheal content of myosin light chain kinase (MLCK), a key enzyme involved in the contraction-relaxation process.

\section{MATERIALS AND METHODS}

\section{TSM preparation}

Experiments were conducted with highly inbred male rats obtained from Charles River (Sulzfeld, Germany). Care of the animals conformed to the recommendations of the Helsinki convention and the study was approved by our institution (INSERM, Paris, France). For mechanical experiments, 10 adult Fisher F-344 rats (means \pm SD weight $208 \pm 38 \mathrm{~g} ; 160-250 \mathrm{~g}$ ) and 10 adult Lewis rats (weight $215 \pm 46 \mathrm{~g} ; 170-280 \mathrm{~g} ; \mathrm{p}=0.71$ ) were studied after being housed at a conventional animal facility where all animals had ad libitum access to food and water. At the time of the experiment, the investigator was not aware of which strain was Fisher or Lewis. For each animal, experiments were performed on TSM obtained from a tracheal ring consisting of four segments. The ring was opened with a dorsal midline section through the cartilage in order to obtain a strip of the posterior membranous portion of the trachea. The epithelium was not removed. The strip was then vertically suspended in a $100 \mathrm{~mL}$ organ bath containing a KrebsHenseleit solution, bubbled with $95 \% \mathrm{O}_{2}$ and $5 \% \mathrm{CO}_{2}$ and maintained at $37^{\circ} \mathrm{C}$, pH 7.4. Composition of Krebs-Henseleit solution was as follows: $118 \mathrm{mM} \mathrm{NaCl}, 4.7 \mathrm{mM} \mathrm{KCl}, 1.2 \mathrm{mM}$ $\mathrm{MgSO}_{4}, 1.2 \mathrm{mM} \mathrm{KH}_{2} \mathrm{PO}_{4}, 25 \mathrm{mM} \mathrm{NaHCO} 3,2.5 \mathrm{mM} \mathrm{CaCl}_{2}$ and $5.5 \mathrm{mM}$ glucose. The lower end of the strip was held by a stationary clip at the bottom of the bath, while the upper extremity was held in a spring clip linked to an electromagnetic lever system. Supramaximal electrical field stimulation (EFS; $30 \mathrm{~V} \cdot \mathrm{cm}^{-1}, 50 \mathrm{~Hz}$ alternating current, $3 \mathrm{~ms}$ pulse duration and $12 \mathrm{~s}$ train duration) was applied every $4 \mathrm{~min}$ by means of two platinum electrodes arranged in parallel on either side of the muscle preparation. Mechanical experiments were conducted after a $1 \mathrm{~h}$ equilibration period.

\section{Electromagnetic lever system}

The electromagnetic device has been described previously [14]. Briefly, an aluminum lever is cemented to a coil suspended in the strong field of a permanent magnet and a force couple develops when an electric current passes through the coil. Force measurement amplitude ranges from 0 to $140 \mathrm{mN}$. The error in measured force is $<0.1 \%$. The equivalent moving mass of the whole system is $155 \mathrm{mg}$ and its compliance is $0.2 \mu \mathrm{m} \cdot \mathrm{mN}^{-1}$. Lever displacement is measured by means of a photoelectric transducer comprising an incandescent lamp, a miniature photodiode and a pre-amplifier acting as a currentto-voltage converter. The light emitted by the lamp is modulated by the displacement of the lever, and current alterations in the photodiode are converted into voltage alterations. System linearity ranges from 0 to $5 \mathrm{~mm}$ of muscle shortening. The error in measured displacement is $<0.5 \%$ of the full-scale deflection.

All analyses were based on digital recordings obtained by means of a personal computer. Two signals, force and length, were recorded simultaneously. The recording speed was one analog-to-digital conversion of each signal every $1 \mathrm{~ms}$. Total recording time ranged from 45 to $60 \mathrm{~s}$. A program developed in our laboratory was used to calculate the mechanical parameters.

\section{Mechanical parameters}

Mechanical parameters were recorded at optimal initial muscle length (LO). For the current study, Lo was defined as the resting length at which the peak value of active isometric tension was measured. It was obtained after successive measurements of isometric tension against increasing preloads. Once determined, the preload corresponding to Lo remained constant throughout the study and was simply called preload. That preload was similar in both TSM strains; $5.3 \pm 2.0 \mathrm{mN} \cdot \mathrm{mm}^{-2}$ in Fisher rats versus $5.7 \pm 2.2 \mathrm{mN} \cdot \mathrm{mm}^{-2}$ in Lewis rats $(\mathrm{p}=0.69)$. It represented $35 \pm 12 \%$ of peak isometric tension in Fisher and $39 \pm 13 \%$ of peak isometric tension in Lewis rats $(p=0.43)$. Five to eight EFSelicited contractions were then performed against increasing loads, from preload up to the fully isometric contraction, according to the conventional afterloaded technique and as previously described [7]. After cessation of the electrical stimulus, we measured the maximum lengthening velocity at preload (Vlength; LO $\cdot \mathrm{s}^{-1}$ ) and peak rate of isometric tension decline $\left(-\mathrm{dP} ; \mathrm{mN} \cdot \mathrm{s}^{-1} \cdot \mathrm{mm}^{-2}\right)$ (fig. 1). During isometric tension decline, the time needed for the muscle to relax from peak of isometric tension to one-half of its active tension ( $t 12$,isom,relax; $\mathrm{s})$ was measured. During isotonic relaxation, half time for relaxation $\left(t \frac{1}{2}\right.$, isot,relax; $\left.s\right)$, which represents the time needed for the muscle to re-elongate from peak of shortening to one-half of its shortening, was measured at all afterload levels (fig. 1) [12, 15]. Using data from the preceding contractile phase, we also calculated the ratio of maximum velocity of shortening at preload (Vshort) to Vlength (Vshort/Vlength) and the ratio of positive to negative peak rate of isometric tension $(+\mathrm{dP} /-\mathrm{dP})$, both indices of mechanical coupling between contraction and relaxation [16]. 
a)

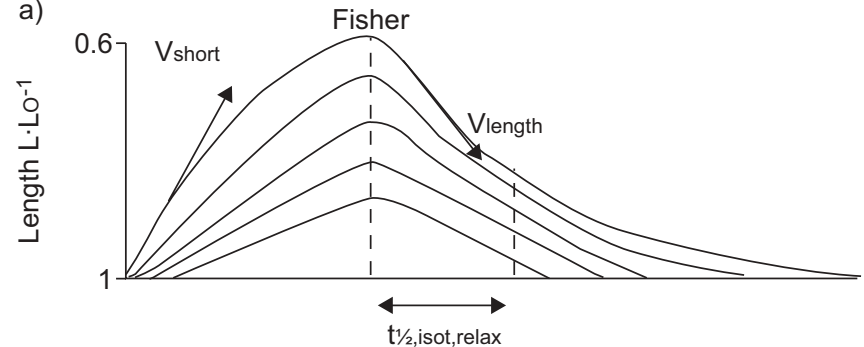

c)

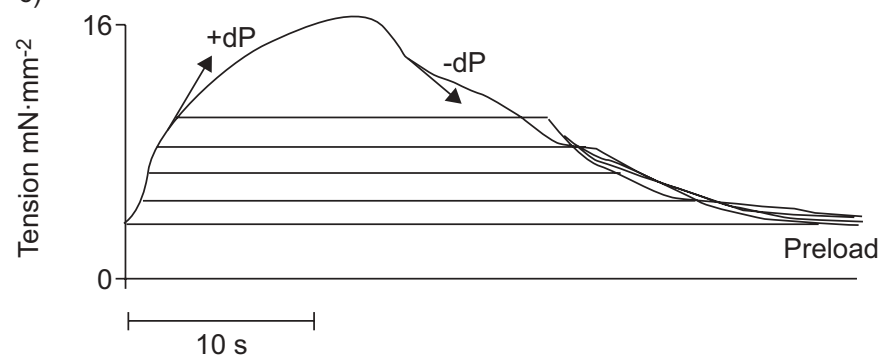

b)

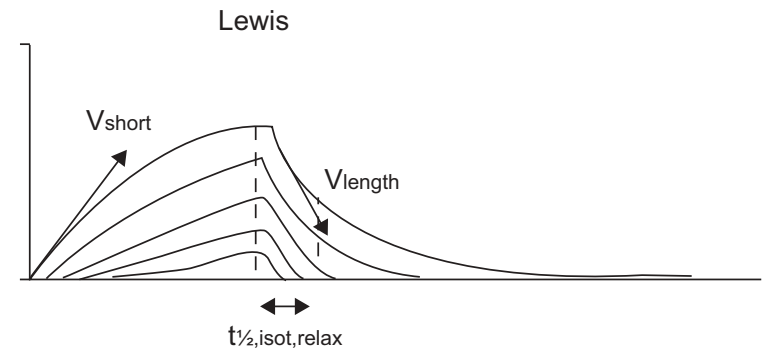

d)

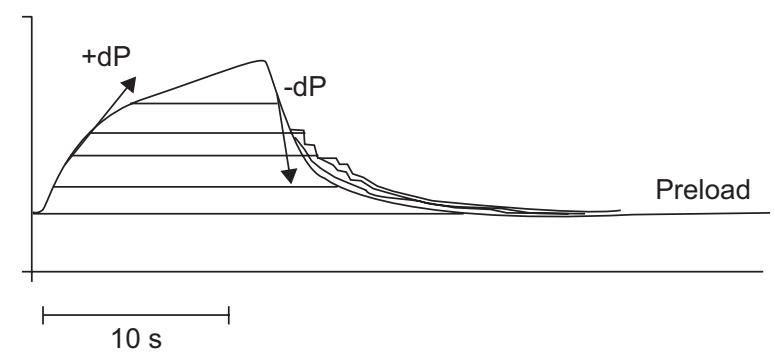

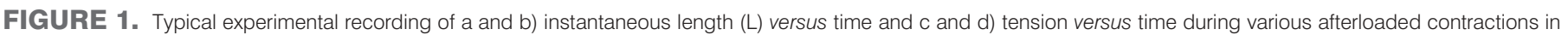

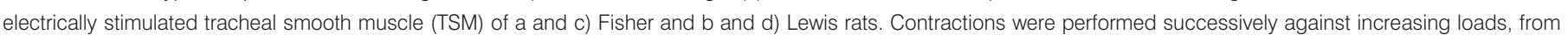

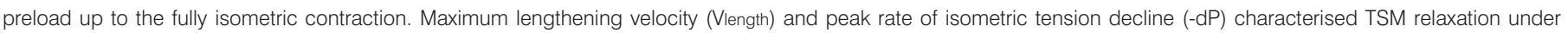

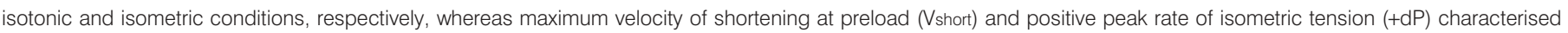

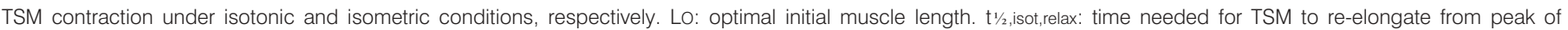
shortening to one-half of shortening.

\section{Measurement of MLCK protein expression}

For MLCK measurements, the whole trachea of three additional Fisher F-344 and four Lewis rats was removed, frozen in liquid nitrogen and stored at $-80^{\circ} \mathrm{C}$ until use. Western blotting was performed on total protein extracts. In brief, muscles were homogenised in SDS buffer containing 1\% SDS, $1 \mathrm{mM}$ sodium vanadate and $10 \mathrm{mM}$ Tris $/ \mathrm{HCl}(\mathrm{pH} \mathrm{7.4)}$ plus a mini-complete protease inhibitor mixture tablet (Roche Molecular Biomedicals, Meylan, France). Protein concentrations were determined using NanoDrop (ND-1000 Spectrophotometer; Thermo Scientific, Wilmington, DE, USA). The samples were stored at $-20^{\circ} \mathrm{C}$ until use. Proteins $(15 \mu \mathrm{g})$ were separated by 9\% SDS-polyacrylamide gel electrophoresis and transferred to nitrocellulose. Membranes were blocked overnight at $4{ }^{\circ} \mathrm{C}$ with $5 \%$ nonfat dry milk in Tween Tris buffered saline (TTBS; $20 \mathrm{mM}$ Tris/ $\mathrm{HCl}, \mathrm{pH} 7.5,140 \mathrm{mM} \mathrm{NaCl}$ and $0.1 \%$ Tween 20). The blots were incubated with goat polyclonal anti-MLCK (L18:SC9452; Tebu-Bio, Le Perray en Yvelines, France) diluted 1:500 in TTBS with 5\% nonfat milk. Immunodetection was performed using anti-goat horseradish peroxidase labelled antibodies diluted 1:5000 in TTBS. The membranes were revealed with enhanced chemiluminescent substrate (GEHealthcare, Orsay, France). Light emission was detected with a highly sensitive imaging system (Fujifilm LAS-3000, Fujifilm Co., St Quentin en Yvelines Cedex, France). Signals were quantified using Image Gauge software (Fuji Photo Film Co., Tokyo Japan) and normalised to the expression of total proteins on Ponceau Red (Sigma-Aldrich, St Quentin Fallavier Cedex, France).

\section{Statistical analysis}

Data are expressed as means $\pm \mathrm{SD}$. Statistical comparisons between strains were carried out using an unpaired t-test.
Correlation between two variables was calculated by linear regression using the least-squares method. A p-value $<0.05$ was considered statistically significant. All p-values were twotailed.

\section{RESULTS}

\section{Isometric and isotonic relaxation}

In both strains, there was no phase of tension decrease below preload during isotonic or isometric relaxation. However, figure 1 illustrates the fact that both isotonic and isometric relaxation markedly differed between Fisher and Lewis rats.

Regarding isotonic relaxation, Vlength was markedly lower in Fisher rats than in Lewis rats $(0.044 \pm 0.019$ and $0.067 \pm 0.021 \mathrm{LO} \cdot \mathrm{s}^{-1}$, respectively; $\mathrm{p}<0.05$ ) (fig. 2). At preload, $\mathrm{t} 1 / 2$, isot,relax was more than doubled in Fisher rats when compared with Lewis rats $(8.33 \pm 3.21$ versus $3.53 \pm 0.54 \mathrm{~s}$; $\mathrm{p}<0.001$ ) (fig. 3). For each studied load ranges, $t 1 \frac{1}{2}$, isot,relax was also markedly prolonged in Fisher than Lewis rats (table 1).

Regarding isometric relaxation, $t^{1} \frac{1}{2}$, isom, relax was markedly prolonged in Fisher rats (fig. 3). In Fisher rats, -dP was also markedly lower $\left(1.62 \pm 1.04 \mathrm{mN} \cdot \mathrm{s}^{-1} \cdot \mathrm{mm}^{-2}\right)$ compared with Lewis rats $\left(3.23 \pm 2.00 \mathrm{mN} \cdot \mathrm{s}^{-1} \cdot \mathrm{mm}^{-2} ; \mathrm{p}<0.05\right.$; fig. 2$)$.

\section{Contraction-relaxation mechanical coupling}

Vshort was $0.072 \pm 0.034 \mathrm{LO} \cdot \mathrm{s}^{-1}$ in Fisher rats and $0.044 \pm$ $0.018 \mathrm{LO} \cdot \mathrm{s}^{-1}$ in Lewis rats $(\mathrm{p}<0.05)$. The Vshort/Vlength ratio was around three times higher in Fisher rats than Lewis rats with a ratio towards two in Fisher while inferior to one in Lewis rats (fig. 4). When Fisher and Lewis rats were taken altogether, there was a positive linear relationship between $t \frac{1}{2}$,isot,relax at preload and the Vshort/Vlength ratio $\left(r^{2}=0.914 ; p<0.0001\right)$ (fig. 5). 

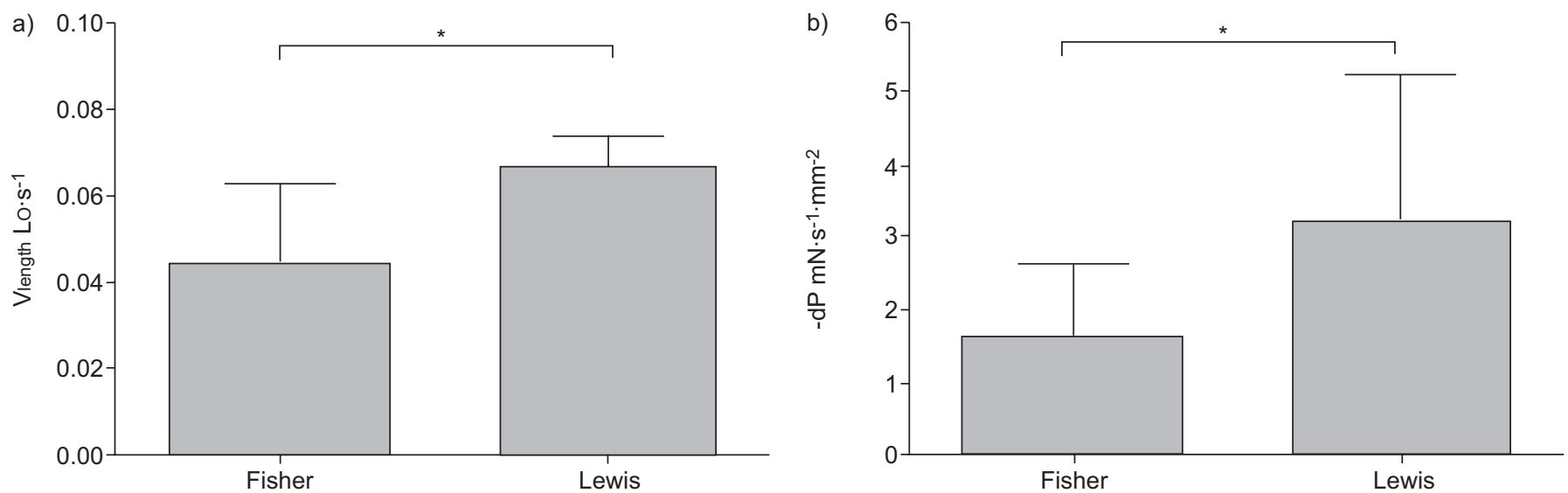

FIGURE 2. Mechanical parameters characterising a) isotonic and b) isometric spontaneous relaxation of electrically stimulated tracheal smooth muscle in Fisher and Lewis rats. Vlength: maximum lengthening velocity at preload; LO: optimal initial muscle length; -dP: peak rate of isometric tension decline derivative. Values are mean \pm SD. *: $p<0.05$

$$
\mathrm{t} 1 / 2, \text { isot,relax }=1.878+3.096(\text { Vshort } / \text { Vlength })
$$

Positive peak rate of isometric tension derivative was $2.68 \pm 1.78 \mathrm{mN} \cdot \mathrm{s}^{-1} \cdot \mathrm{mm}^{-2}$ in Fisher rats and $2.18 \pm$ $1.31 \mathrm{mN} \cdot \mathrm{s}^{-1} \cdot \mathrm{mm}^{-2}$ in Lewis rats $(\mathrm{p}=0.48)$. The $+\mathrm{dP} /-\mathrm{dP}$ ratio was approximately three times higher in Fisher rats than in Lewis rats with a ratio slightly superior to two in Fisher while inferior to one in Lewis rats (fig. 4).

\section{MLCK protein expression}

Representative Western-blots from Fisher and Lewis rats' tracheal muscles are presented in figure 6a. When normalised to the expression of total proteins on Ponceau Red, signals of MLCK were markedly decreased in Lewis rats compared to Fisher rats (fig. 6b). The amount of MLCK was nearly six-fold lower in Lewis rats than in Fisher rats $(p<0.01)$ (fig. 6c).

\section{DISCUSSION}

The major finding of the present study is that Fisher and Lewis rat strains markedly differed from each other when considering both isotonic and isometric spontaneous relaxation of their electrically stimulated TSM. In Fisher rats, relaxation was prolonged and slowed throughout the load continuum when compared with Lewis rats. Analysis of the contraction-relaxation coupling revealed that contraction was favoured in Fisher rats whereas relaxation was prevalent in Lewis rats. Such mechanical differences were associated with a lower amount of MLCK in the TSM of Lewis rats.

\section{The "Fisher/Lewis" model}

Both Fisher and Lewis rats are anatomically well-characterised strains of inbred rats [17]. When compared with Lewis rats, Fisher rats are known to exhibit higher airway responsiveness to various inhaled contractile agonists such as methacholine [2] or 5-hydroxytryptamine $[3,4]$. In vitro, the ASM of these two rat strains exhibits marked differences: while isolated TSM develops similar peak isometric force in both strains [2,7], the TSM of Fisher rats has been shown to be more responsive to cumulative concentrations of carbachol [18] or serotonin [19] than the Lewis
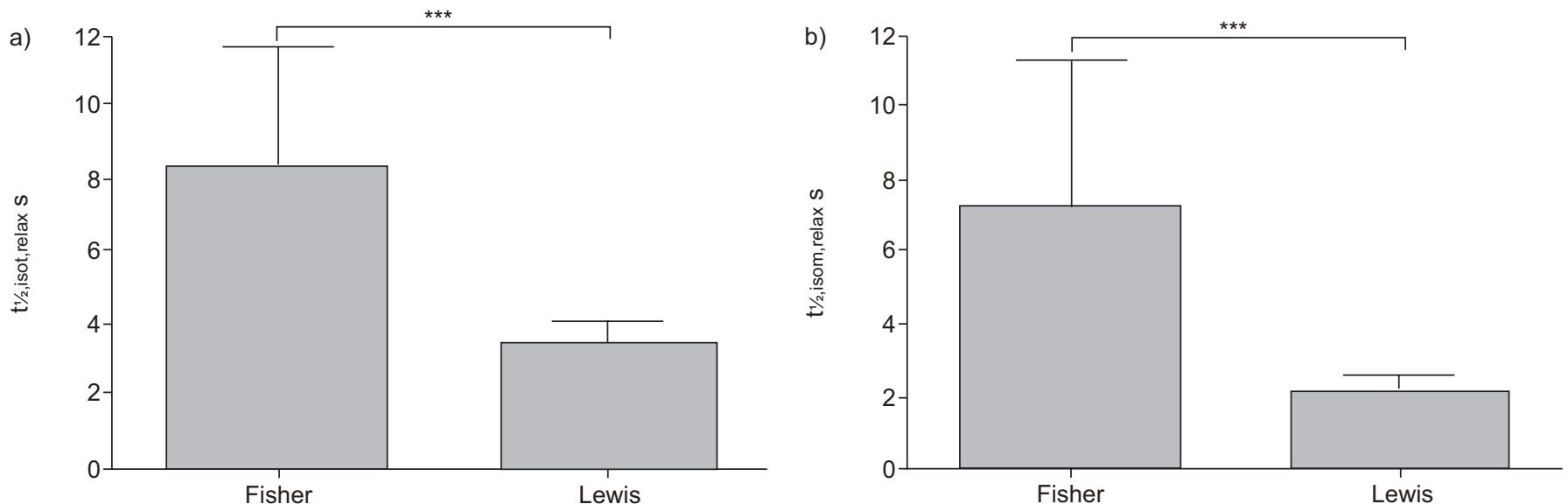

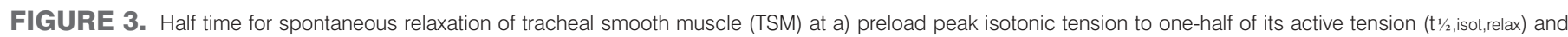

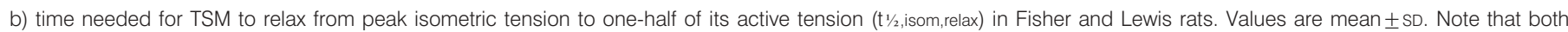

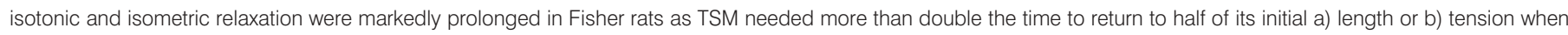
compared with Lewis rats. ${ }^{* * *}$ : $p<0.001$. 


\begin{tabular}{|c|c|c|c|c|}
\hline \multirow{3}{*}{\multicolumn{2}{|c|}{$\begin{array}{l}\text { TABLE } 1 \text { Ha } \\
\text { at } \\
\text { Load \% isometric } \\
\text { tension }\end{array}$}} & \multicolumn{3}{|c|}{$\begin{array}{l}\text { Half time for tracheal smooth muscle relaxation } \\
\text { at several load ranges in Fisher and Lewis rats }\end{array}$} \\
\hline & & \multicolumn{2}{|c|}{$t / 2$, isot, relax $S$} & \multirow{2}{*}{$p$-value } \\
\hline & & Fisher & Lewis & \\
\hline \multicolumn{2}{|l|}{$15-40$} & $7.89 \pm 1.90$ & $3.05 \pm 0.50$ & $<0.0001$ \\
\hline \multicolumn{2}{|l|}{$40-60$} & $6.86 \pm 2.98$ & $2.51 \pm 0.89$ & 0.0001 \\
\hline \multicolumn{2}{|l|}{$60-90$} & $4.84 \pm 3.72$ & $1.58 \pm 0.56$ & $<0.005$ \\
\hline
\end{tabular}

strain. Lung explants have been used to show that this innate relative hyperresponsiveness of the Fisher strain was not restricted to the trachea but extended throughout the overall airway tree [4, 20]. Finally, it has been recently shown that electrically stimulated TSM of Fisher rats exhibited higher shortening capacity and velocity than in Lewis rats [7]. Taken together, these results support the hypothesis that the relative nonspecific AHR found in the Fisher strain could be related to differences in the intrinsic contractile properties of the ASM [4].

\section{Relaxation in AHR}

Surprisingly, isotonic relaxation of smooth muscle has been rarely investigated in animal models of AHR. In ragweed pollen sensitised, electrically stimulated canine bronchial smooth muscle, JIANG and STEPHENS [12] have shown that isotonic relaxation was prolonged ( $83 \%$ increased) after $1 \mathrm{~s}$ of stimulation when compared with that of muscle from litter mate controls. These differences were abolished when measuring the same mechanical parameters after $10 \mathrm{~s}$ of stimulation. As relaxation time of latch bridges was not different between sensitised bronchi and controls, this indicates alterations in properties of the sole early recruited (within the first $2 \mathrm{~s}$ ) normally cycling crossbridges [13]. Similar results have been obtained in electrically stimulated canine venous muscle strips [15].

In guinea-pigs, CHITANO and co-workers [10, 21] have shown that isometric relaxation of electrically stimulated TSM is decreased in 1-week-old animals compared with 3-week and 3month-old animals while calculated maximum shortening velocity extrapolated at zero-load is reduced in adults. These results suggest that increased velocity of shortening and decreased isometric relaxing capacity could contribute, at least in part, to the greater incidence of AHR reported in children and juvenile animals [8]. It should be noted that maturational changes have also been reported in smooth muscle mechanics, especially in vascular pulmonary and systemic (carotid artery) smooth muscle of sheep: isotonic relaxation was decreased in adults compared with newborns [22]. Despite these few studies, no data are available concerning isotonic and isometric relaxation of ASM in animal models of spontaneous inherited, genetically determined AHR or in human asthmatics.

\section{Isotonic relaxation in Fisher and Lewis rats}

Isotonic relaxation was slowed and prolonged in Fisher rats when compared to Lewis rats, as attested by lower Vlength and longer $t \frac{1}{2}$, isot,relax values (table 1 and figs 2 and 3). During isotonic relaxation, mechanisms working to reduce crossbridge interactions are opposed by mechanical forces that tend to produce relative myofilament shearing as the muscle returns to its resting length [23]. Our study showed that whatever the load facing TSM during contraction, the time required in Fisher rats to re-elongate from the peak of TSM shortening to one-half of its shortening more than doubled when compared with what occurred in Lewis rats (table 1). It also indicates that $t 1 / 2$,isot,relax values were highly dependent on the load facing the muscle: the higher the load, the shorter the extent of muscle shortening and the shorter the $t \frac{1}{2}$, isot,relax value.

In the conditions under study, the lack of any tension decrease below preload level during isotonic, and isometric, relaxation indicated that, in both rat strains, there was no active resting tension (or active intrinsic tone). Thus, from a mechanical point of view, the electrically stimulated TSM of rats behaves like canine [24, 25], porcine [26], mouse [27] or rabbit [28] TSM and differs from bronchial smooth muscle of humans [14] and TSM of guinea-pigs $[10,29]$, which are both known to exhibit active resting tension. The present data clearly indicate that innate differences in airway responsiveness could not be attributed to differences in active resting tension level in the rat strains under study.

The finding that TSM isotonic relaxation was markedly prolonged in Fisher rats is of particular importance. In fact, when the time course of relaxation is prolonged, TSM needs more time to return to resting length and tension. Processes that lead to the disappearance of force-generating sites are less effective: impairment of the TSM ability to return to resting conditions can contribute, at least in part, to the in vivo relative AHR of Fisher rats. In Fisher rats, a slower rate of relaxation could prolong the effects of airways constriction, delaying their reduction and elimination.

\section{Isometric relaxation in Fisher and Lewis rats}

As was the case for ASM in other species' including humans [14], isometric relaxation of TSM was of the "load-independent" type in both strains of rats under study: time course of isometric relaxation was not affected by the afterload level. Load-independent relaxation is typical of sarcomeric muscles in which the endoplasmic reticulum is poorly developed, nonfunctional, destroyed or inhibited [30]. It is also obviously the case in ASM where endoplasmic reticulum exists only in a rudimentary form [31]. This mechanical property should be distinguished from what happens during isotonic relaxation where $t \frac{1}{2}$,isot,relax values are markedly influenced by the level of load (table 1).

In the present study, isometric relaxation was markedly decreased in Fisher rats when compared with Lewis rats, as attested by lower - $\mathrm{dP}$ values (fig. 2). Such a difference is remarkable when one considers that both strains exhibit the same level of maximal isometric tension [7]. Mechanisms involved in such a finding remain to be precisely determined but some hypotheses can be made. In smooth muscle, the decline of isometric tension is known to be associated with a decrease in the calcium bound to calmodulin, usually as a result of the resequestration of internal calcium or the active extrusion of cytosolic calcium [23]. This decrease in intracellular calcium 

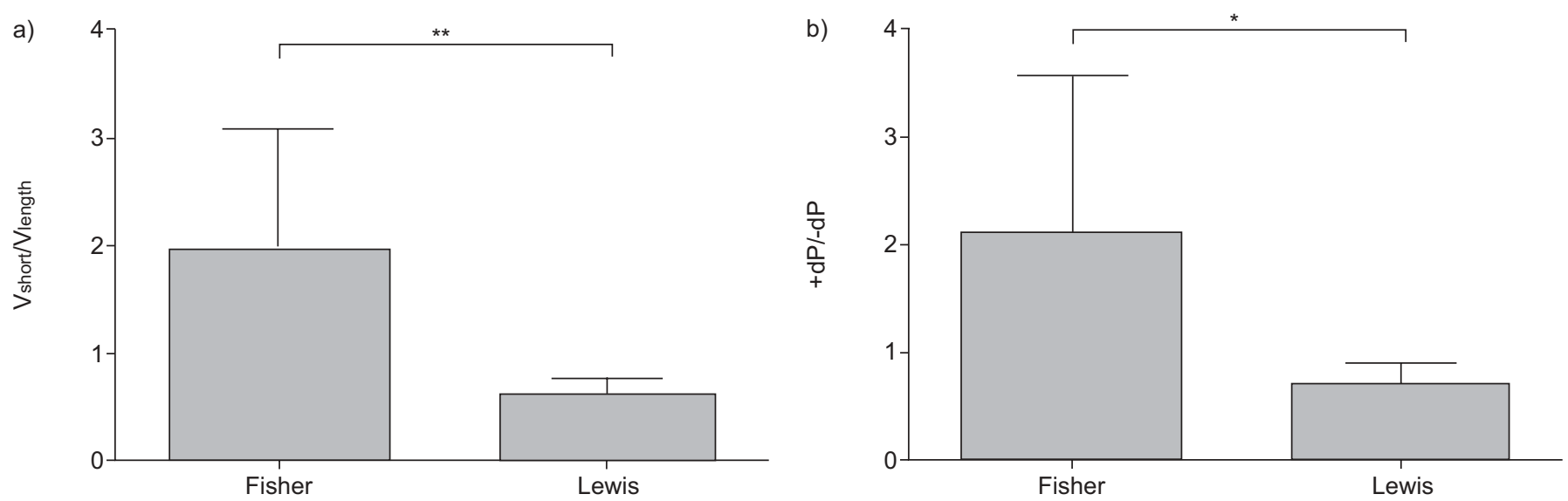

FIGURE 4. Indices characterising mechanical coupling between contraction and relaxation of tracheal smooth muscle in a) isotonic and b) isometric conditions in Fisher and Lewis rats. Vshort: maximum shortening velocity at preload; Vlength: maximum lengthening velocity at preload; +dP: positive peak rate of isometric tension derivative; -dP negative peak rate of isometric tension derivative. Values are mean $\pm S D$. ${ }^{*}: p<0.05 ;{ }^{*}: p<0.01$.

level leads to a decline in the rate at which the $20-\mathrm{kD}$ regulatory light chains of myosin are phosphorylated. Myosin light chain phosphatase activity predominates over MLCK. In the present study, MLCK tracheal content was found to be markedly higher in Fisher rats than in Lewis rats (fig. 6), indicating that one of the most important enzymes required to produce force could contribute to the sustained TSM contraction observed in Fisher rats. According to this hypothesis, increased MLCK would favour the contraction in Fisher rats and be associated with an impairment of relaxation. Other intracellular mechanisms resulting in an increase of cytosolic calcium and/or a decrease in myosin light chain phosphatase activity may also contribute to delaying the time course of muscle relaxation. Potential candidates not explored in this study are: 1) differences in the structure, content or activity of myosin light chain phosphatase; 2) structural or functional differences in the $20-\mathrm{kD}$ regulatory light chains of myosin; 3) differences in the

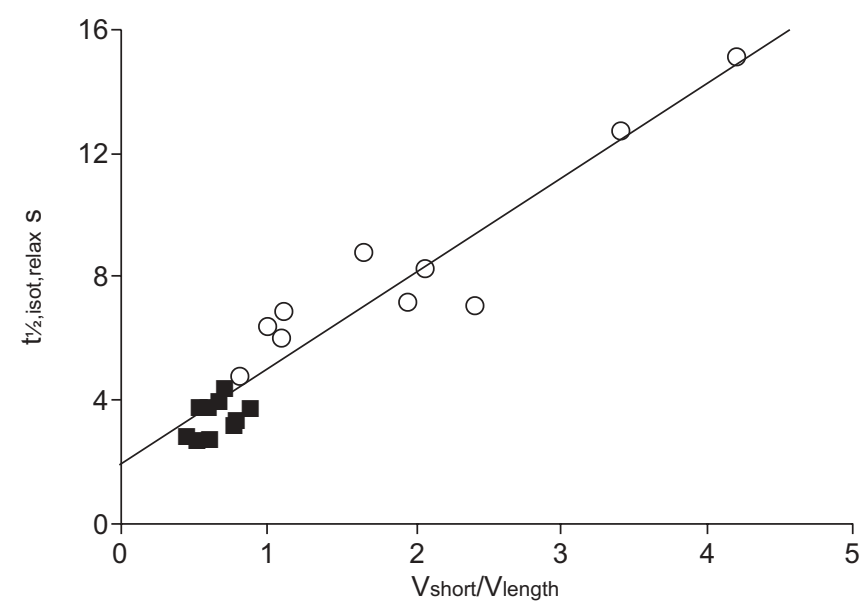

FIGURE 5. Relationship between the ratio of maximum shortening velocity to maximum lengthening velocity at preload (Vshort and Vlength, respectively) of tracheal smooth muscle (TSM) and the half time for TSM relaxation at preload

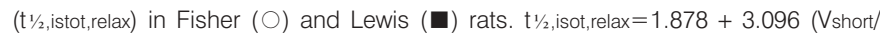
Vlength). $r^{2}=0.914, p<0.0001$. activity of the Rho-associated kinase that can directly phosphorylate the $20-\mathrm{kD}$ regulatory light chains of myosin independently of calcium [32]; and 4) structural or functional differences in the calcium-calmodulin complex. Among others, these potential mechanisms have also been proposed to contribute, at least in part, to the higher TSM shortening capacity and velocity in Fisher rats associated with a shorter duration of crossbridge detachment and attachment [7]. It should be mentioned that increased intracellular calcium mobilisation has been described in Fisher rats when compared with Lewis rats [4, 33], suggesting that calcium-dependent pathways were at least partly involved in the mechanisms underlying the nonspecific AHR of the Fisher strain.

\section{Contraction-relaxation coupling in Fisher and Lewis rats}

We have previously demonstrated that isolated TSM of Fisher rats exhibited higher maximum unloaded shortening velocity and increased extent of muscle shortening without any differences in peak isometric tension when compared with Lewis rats [7]. In the present study, we found that both the Vshort/Vlength ratio and the $+\mathrm{dP} /-\mathrm{dP}$ ratio were approximately three times higher in Fisher rats than in Lewis rats (fig. 4). These ratios were close to two in Fisher rats while inferior to one in Lewis rats. From a mechanical point of view, these results indicate that contraction was favoured in Fisher rats whereas relaxation was prevalent in Lewis rats. In fact, as velocities of muscle shortening were higher in Fisher rats than in Lewis rats, one could have expected that lengthening velocity would also be higher in Fisher rats, leading to a Vshort/Vlength ratio towards one. This was clearly not the case, leading to a kind of "overall amplification" of the higher rate of the contraction of TSM of Fisher rats in isotonic mode, contributing to maintain the exaggerated muscle shortening that is a key feature of AHR. Moreover, as there were no interstrain differences regarding peak isometric tension and the positive peak of its derivative, one could have expected no differences in the negative peak rate of isometric tension derivative that helps to characterise isometric relaxation. Again, this was not the case as $-\mathrm{dP}$ was lower in Fisher rats than in Lewis rats, leading to $\mathrm{a}+\mathrm{dP} /-\mathrm{dP}$ ratio close to a value of two. Thus, despite a similar isometric contraction, the TSM 
a)

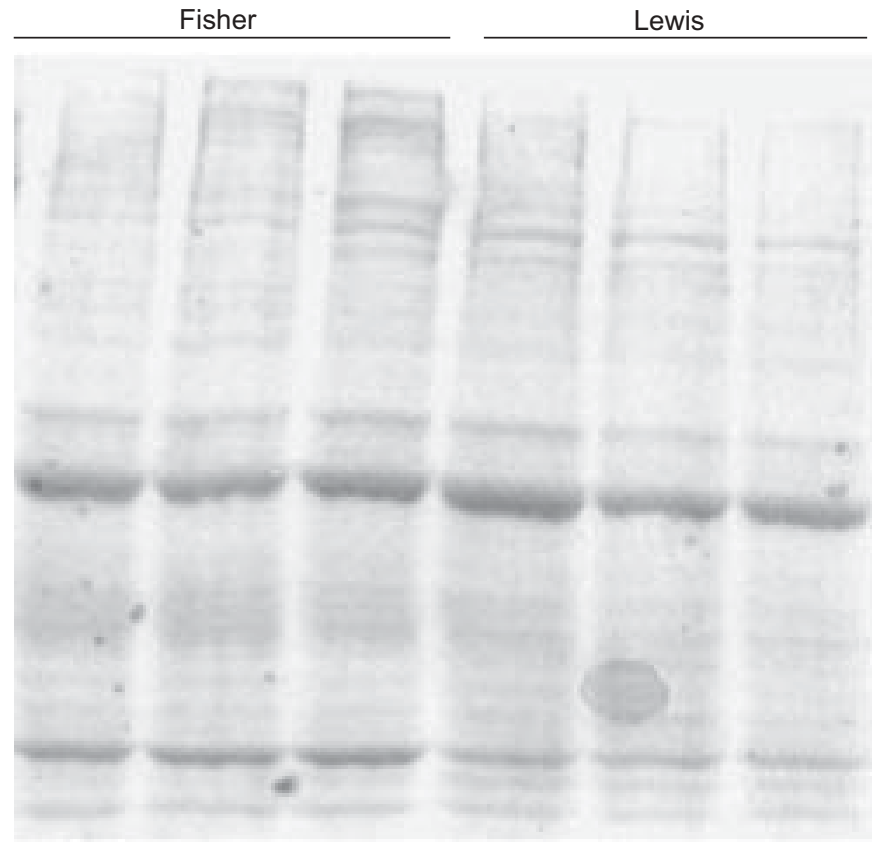

b)

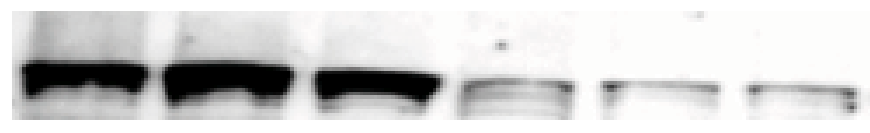

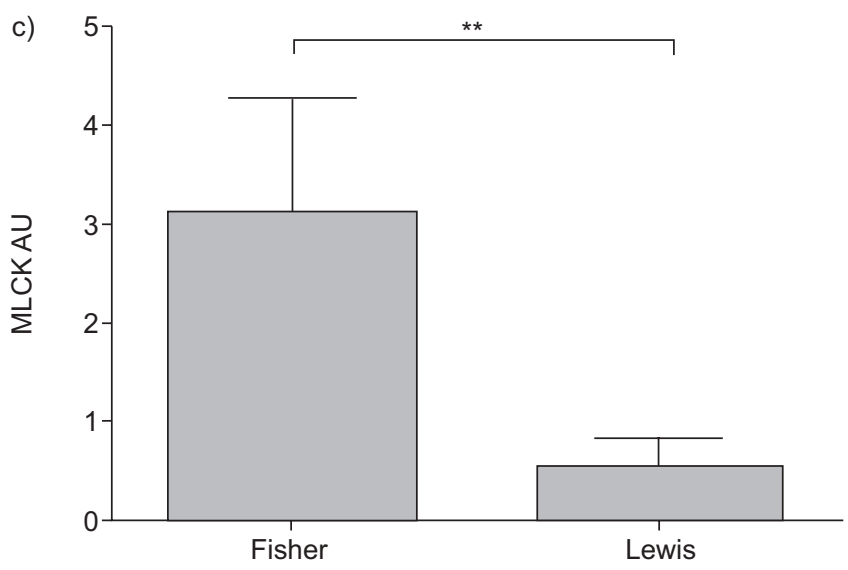

FIGURE 6. Myosin light chain kinase (MLCK) expression in Fisher and Lewis rats' tracheal smooth muscles. a) Typical Ponceau red and b) immunoblots at $160 \mathrm{kD}$ obtained with specific antibodies directed against MLCK. C) MLCK quantifications normalised to the expression of total proteins on Ponceau red were reported in arbitrary units (AU). Values are mean $\pm S D, n=3$ in Fisher rats and $n=4$ in Lewis rats. ${ }^{* *}: \mathrm{p}<0.01$.

asthmatic airways. However, when contractility and pharmacological relaxation are investigated, results can be somewhat conflicting when the contractile response of isolated asthmatic ASM is considered $[35,36]$. In asthma, a reduced relaxation response to various bronchodilating agents has been observed in both tracheal and bronchial ASM, leading to the idea that asthmatic ASM has abnormalities in relaxation response rather than contractile response [37]. The finding that spontaneous relaxation is also impaired in a model of spontaneous, inherited, genetically determined AHR is of particular importance in that context. In fact, if such an impairment would be observed in ASM of asthmatics, which remains to be proven, the magnitude of the residual failure to relax could account, at least in part, for the magnitude of bronchospasm, as previously hypothesised [38]. One of the consequences of our results is that they emphasise the importance and the relevance of further studies focusing on isotonic ASM relaxation in patients who suffer from asthma [38]. Further studies should also be designed to investigate molecular mechanisms associated with our findings, especially at the crossbridge level. Finally, the balance between MLCK and myosin light chain phosphatase deserves further attention.

In conclusion, we analysed the spontaneous isotonic and isometric relaxation of isolated TSM of the Fisher and Lewis inbred rat strains. We found that relaxation was slowed and prolonged in Fisher rats as TSM needed more than double the time to return to half of its initial length or tension when compared with Lewis rats. In this model of innate greater airway responsiveness characterising the Fisher rat strain, both reduced level and slower rate of relaxation attested to a global impairment of the ability of electrically stimulated TSM to relax in isotonic and isometric ways. Such an impairment could contribute to facilitate the maintenance of the exaggerated 
airway constriction that is associated with the previously reported increase in TSM shortening capacity and velocity and differences in myosin crossbridge kinetics. One of the potential mechanisms involved in such mechanical differences is a markedly increased amount of MLCK found in the TSM of Fisher rats when compared with Lewis rats. Taken together, these findings highlight the crucial role of ASM in the phenomenon of innate greater airway responsiveness of the Fisher rat strain.

\section{STATEMENT OF INTEREST}

None declared.

\section{REFERENCES}

1 Brusasco V, Crimi E, Pellegrino R. Airway hyperresponsiveness in asthma: not just a matter of airway inflammation. Thorax 1998; 53: 992-998.

2 Di Maria GU, Martin JG, Bellofiore S, et al. Relationship between in vivo airway reactivity and in vitro responsiveness of tracheal smooth muscle in inbred rats. Respiration 1988; 54: Suppl. 1, 108-113.

3 Zacour ME, Martin JG. Enhanced growth response of airway smooth muscle in inbred rats with airway responsiveness. Am J Respir Cell Mol Biol 1996; 15: 590-599.

4 Tao FC, Tolloczko B, Eidelman DH, et al. Enhanced $\mathrm{Ca}^{2+}$ mobilization in airway smooth muscle contributes to airway hyperresponsiveness in an inbred strain of rat. Am J Respir Crit Care Med 1999; 160: 446-453.

5 Dandurand R, Xu L, Martin J, et al. Airway parenchymal interdependence and bronchial responsiveness in two highly inbred rat strains. J Appl Physiol 1993; 74: 538-544.

6 Shore SA. Airway smooth muscle in asthma - not just more of the same. N Engl J Med 2004; 351: 531-532.

7 Blanc FX, Coirault C, Salmeron S, et al. Mechanics and crossbridge kinetics of tracheal smooth muscle in two inbred rat strains. Eur Respir J 2003; 22: 227-234.

8 Chitano P, Murphy TM. Maturational changes in airway smooth muscle shortening and relaxation. Implications for asthma. Respir Physiol Neurobiol 2003; 137: 347-359.

9 Hill AV. The heat of shortening and the dynamic constants of muscle. Proc R Soc Lond B Biol Sci 1938; 126: 136-195.

10 Chitano P, Cox CM, Murphy TM. Relaxation of guinea pig trachealis during electrical field stimulation increases with age. J Appl Physiol 2002; 92: 1835-1842.

11 Knox AJ, Tattersfield AE. Airway smooth muscle relaxation. Thorax 1995; 50: 894-901.

12 Jiang H, Stephens NL. Isotonic relaxation of sensitized bronchial smooth muscle. Am J Physiol 1992; 262: L344-L350.

13 Stephens NL, Jiang H. Relaxation of smooth muscle. Can J Physiol Pharmacol 1994; 72: 1345-1350.

14 Blanc FX, Salmeron S, Coirault C, et al. Effects of load and tone on the mechanics of isolated human bronchial smooth muscle. J Appl Physiol 1999; 86: 488-495.

15 Liu X, Jiang H, Stephens NL. Use of a new index to study relaxation in a vascular model of anaphylactic shock. J Appl Physiol 1993; 74: 2621-2626.

16 Péry-Man N, Chemla D, Coirault C, et al. A comparison of cyclopiazonic acid and ryanodine effects on cardiac muscle relaxation. Am J Physiol 1993; 265: H1364-H1372.

17 Riesenfeld A. Constitution and body proportions in different strains of rats. Acta Anat 1976; 94: 169-183.
18 Jia $Y, X u$ L, Heisler S, et al. Airways of a hyperresponsive rat strain show decreased relaxant responses to sodium nitroprusside. Am J Physiol Lung Cell Mol Physiol 1995; 269: L85-L91.

19 Florio C, Styhler A, Heisler S, et al. Mechanical responses of tracheal tissue in vitro: dependence on the tissue preparation employed and relationship to smooth muscle content. Pulm Pharmacol 1996; 9: 157-166.

20 Wang CG, Almirall JJ, Dolman CS, et al. In vitro bronchial responsiveness in two highly inbred rat strains. J Appl Physiol 1997; 82: 1445-1452.

21 Chitano P, Wang J, Cox CM, et al. Different ontogeny of rate of force generation and shortening velocity in guinea pig trachealis. J Appl Physiol 2000; 88: 1338-1345.

22 Belik J, Stephens NL. Developmental differences in vascular smooth muscle mechanics in pulmonary and systemic circulations. J Appl Physiol 1993; 74: 682-687.

23 Meiss RA. Mechanics of smooth muscle contraction. In: Kao CY, Carsten ME, eds. Cellular aspects of smooth muscle function. Cambridge, Cambridge University Press; 1997; pp. 169-208.

24 Russell JA. Responses of isolated canine airways to electric stimulation and acetylcholine. J Appl Physiol 1978; 45: 690-698.

25 Antonissen LA, Mitchell RW, Kroeger EA, et al. Mechanical alterations of airway smooth muscle in a canine asthmatic model. J Appl Physiol 1979; 46: 681-687.

26 Ishida K, Paré PD, Blogg T, et al. Effects of elastic loading on porcine trachealis muscle mechanics. J Appl Physiol 1990; 69: 10331039.

27 Fan T, Yang M, Halayko A, et al. Airway responsiveness in two inbred strains of mouse disparate in IgE and IL-4 production. Am J Respir Cell Mol Biol 1997; 17: 156-163.

28 Blanc FX, Langeron O, Coirault C, et al. Mechanical properties of tracheal smooth muscle are impaired in the rabbit with experimental cardiac pressure overload. Chest 2004; 125: 236-242.

29 Bard M, Salmeron S, Coirault C, et al. Effects of initial length on intrinsic tone in guinea pig tracheal smooth muscle. Am J Physiol 1998; 275: L1026-L1030.

30 Lecarpentier $\mathrm{Y}$, Coirault C, Langeron O, et al. Impaired load dependence of diaphragm relaxation during congestive heart failure in the rabbit. J Appl Physiol 1999; 87: 1339-1345.

31 Gabella G. Anatomy of airways smooth muscle. In: Raeburn D, Giembycz MA, eds. Airways Smooth Muscle: Structure, Innervation and Neurotransmission. Boston, Birkäuser Verlag, 1994; pp. 1-27.

32 Kureishi Y, Kobayashi S, Amano M, et al. Rho-associated kinase directly induces smooth muscle contraction through myosin light chain phosphorylation. J Biol Chem 1997; 272: 12257-12260.

33 Tao FC, Tolloczko B, Mitchell CA, et al. Inositol $(1,4,5)$ trisphosphate metabolism and enhanced calcium mobilization in airway smooth muscle of hyperresponsive rats. Am J Respir Cell Mol Biol 2000; 23: 514-520.

34 Ma X, Li W, Stephens NL. Detection of two clusters of mechanical properties of smooth muscle along the airway tree. J Appl Physiol 1996; 80: 857-861.

35 Goldie RG, Spina D, Henry PJ, et al. In vitro responsiveness of human asthmatic bronchus to carbachol, histamine, $\beta$-adrenoceptor agonists and theophylline. Br J Clin Pharmacol 1986; 22: 669-676.

36 Bai TR. Abnormalities in airway smooth muscle in fatal asthma. Am Rev Respir Dis 1990; 141: 552-557.

37 Munakata M. Airway remodeling and airway smooth muscle in asthma. Allergol Int 2006; 55: 235-243.

38 An SS, Bai TR, Bates JHT, et al. Airway smooth muscle dynamics: a common pathway of airway obstruction in asthma. Eur Respir J 2007; 29: 834-860. 